\title{
Fluorescence Spectra of 7,8-Benzoquinoline Isolated in the Supersonic Jet Expansion - An Ab Initio Analysis
}

\author{
I. Deperasińska ${ }^{a, *}$, J. Prochorow ${ }^{a}$ And Y. Stepanenko ${ }^{b}$ \\ ${ }^{a}$ Institute of Physics, Polish Academy of Sciences \\ al. Lotników 32/46, 02-668 Warsaw, Poland \\ ${ }^{b}$ Institute of Physical Chemistry and The Laser Center \\ Polish Academy of Sciences, ul. M. Kasprzaka 44, 01-224 Warsaw, Poland
}

(Received June 30, 2004; in final form August 16, 2004)

The optimized equilibrium geometry of 7,8-benzoquinoline molecule in its first excited, $S_{1}$, singlet state was computed with the use of $a b$ initio RCIS/6-31G(d) method. It was found that the electronic transition to the first excited state in 7,8-benzoquinoline is not confined to the neighborhood of nitrogen atom, but is delocalized over entire aromatic rings system, much alike in the molecule of phenanthrene. With the optimized geometry of the ground and excited state of the molecule, the frequencies of the vibrational fundamental modes were computed, together with their displacement parameters (geometry changes of vibrations between the excited and the ground state). These frequencies are in good agreement with vibrational frequencies present in the fluorescence spectrum of 7,8-benzoquinoline observed recently under jet-cooling conditions in supersonic beam expansion. In comparison to the fluorescence spectrum of phenanthrene, the calculated, as well as experimental fluorescence spectra of 7,8-benzoquinoline contain much more vibrational features, and this increased vibronic activity is related to the symmetry break caused by the introduction of N-heteroatom into the aromatic ring system of phenanthrene.

PACS numbers: 82.20.Wt, 31.15.Ar, 33.20.Tp

*corresponding author; e-mail: deper@ifpan.edu.pl 


\section{Introduction}

There are no simple and strict rules, that could be used for a prediction of the changes of spectral properties of a polycyclic aromatic hydrocarbon molecule upon substitution of a nitrogen atom(s), into the aromatic ring system (and creation in this way its N-heterocyclic aromatic derivative). This is connected, at least in part, with occurrence of a new quality in the $\pi$-electrons aromatic system of aromatic rings, brought about by the presence of non-bonding $n$-electrons of the lone-pair(s) of nitrogen atom(s). In addition to the electronic $\pi, \pi^{*}$ states, characteristic of an aromatic hydrocarbon molecule, the $n, \pi^{*}$ states are occurring within the both, singlet and triplet electronic-excited-states manifolds. These different electronic configurations can mix and couple delivering in this way different routes for radiative and nonradative decays of different excited N-heterocyclic aromatic hydrocarbon molecules (for a review of earlier experimental and theoretical data and findings see $[1,2])$. On top of that, the $n$-electrons, which are non-bonding (from the point of view of the aromatic system), are frequently open to the specific interactions with the molecules of the surroundings, which in the protic solvents may lead to the hydrogen-bond formation or protonation. Hence, in some cases fluorescence and/or phosphorescence of N-heterocyclic aromatic hydrocarbons are extremely sensitive to the solvent. The prime example of such an extreme sensitivity is the molecule of acridine - monoazaanthracene, which is virtually non-fluorescent in non-polar aprotic solvents (such as $n$-hexane) and exhibits a very strong fluorescence in protic, hydroxylic solvents [3].

On the other hand, fluorescence of such monoaza-substituted tricyclic aromatic hydrocarbons as catacondensed monoaza-phenanthrenes - 7,8benzoquinoline (BQ) and phenanthridine, is in non-polar aprotic solvents (as $n$-hexane) more intense, as compared to the fluorescence of their parent hydrocarbon - phenanthrene. In the solution of polar, hydrogen-bonding methanol, fluorescence of both molecules is enhanced, but this enhancement is much larger for phenanthridine than for 7,8-benzoquinoline. It has turned out that this difference is primarily due to a decrease in the rate constant for nonradiative transitions from the $S_{1}$ excited singlet state, which is much larger for phenanthridine than for BQ molecule [4]. An ab initio analysis of such experimental results [5] shows that in both molecules the first excited, $S_{1}$, singlet state is of $\pi \pi^{*}$ character. They also show that the excited ${ }^{1}\left(n, \pi^{*}\right)$ singlet state is the third, $\left(S_{3}\right)$, and the second, $\left(S_{2}\right)$, excited singlet state in BQ and phenanthridine, respectively (within the approximation of ground-state optimized geometry for both molecules). The results of TD DFT B3LYP/6-31G(d) calculations of the $S_{0} \rightarrow S_{n}$ transition energies have shown that the separation between the first excited ${ }^{1}\left(\pi, \pi^{*}\right)$ and the $^{1}\left(n, \pi^{*}\right)$ states in BQ (i.e. $S_{3}-S_{1}$ separation) equals $3614 \mathrm{~cm}^{-1}$, while in phenanthridine this separation (i.e. $S_{2}-S_{1}$ separation) is equal only to $1810 \mathrm{~cm}^{-1}$ [5]. Such a difference in the energy gap between ${ }^{1}\left(\pi, \pi^{*}\right)$ and ${ }^{1}\left(n, \pi^{*}\right)$ excited states in both molecules might be responsible for the difference in the mechanism of enhancement of their 
fluorescence, observed upon going from $n$-hexane to methanol solution, provided the position (energy) of excited ${ }^{1}\left(n, \pi^{*}\right)$ state of each of these molecules is differently altered by the presumable hydrogen bond formation with solvent (methanol) molecules. If this would be the case, one may expect a different modification of the spin-orbit (or spin-orbit vibronic coupling) between the manifolds of excited singlet and triplet states and different changes in the singlet-triplet intersystem crossing nonradiative transitions and in the overal rate for nonradiative transitions from excited singlet state in each of these molecules.

It is clear that for a deeper insight into the above-outlined problem one has to switch from the ground-state to excited-states properties and structures (optimized equilibrium geometry, as well as the electronic structure). Optimization of the geometry of excited singlet state within the framework of $a b$ initio restricted configuration interaction-singles (RCIS) method seems to be the most reliable among the present methods, especially if the results can be compared with a clearly resolved vibronic structure of fluorescence spectra (which is not the case for fluorescence in liquid solutions), and further confirmed by the normal-mode analysis and assignments of vibrations.

In our recent work we have reported the results of the observations of laser induced fluorescence spectra of 7,8-benzoquinoline (and phenanthridine) under jetcooling conditions in a supersonic expansion of helium [6]. And these experimental data, which by virtue are probing the vibrational structure of the ground state, can be used as the reference ground for analysis, discussion, and comparison of the properties of excited singlet state of the molecules under consideration.

In this paper we present the first part of such an analysis, i.e. the results of an $a b$ initio analysis of the fluorescence spectrum of 7,8-benzoquinoline. These results are discussed in comparison with the results of analysis of similar observations of jet-cooled fluorescence spectra for the phenanthrene molecule [7] — parent aromatic hydrocarbon for BQ.

\section{Excited $S_{1}$ singlet state of 7,8-benzoquinoline}

As we mentioned in the preceding section, the optimized geometry of the ground state of BQ molecule has been found and discussed earlier [5]. In the optimization procedure we have utilized both, the restricted Hartree-Fock method at 6-31G(d) level (RHF/6-31G(d)) and the density functional theory at B3LYP/6$-31 \mathrm{G}(\mathrm{d})$ level (DFT B3LYP/6-31G(d)). It was found that optimized geometry has a planar structure, and that the bond lengths determined within the framework of both methods are similar (with difference not exceeding $0.02 \AA$ ). However, they differ from the bond lengths of phenanthrene molecule and this difference is especially pronounced for those bonds of BQ molecule which are formed with participation of $\mathrm{N}$ atom (which replaces $\mathrm{C}$ atom in the phenanthrene's ring system). This in turn leads to the changes of the angles between bonds in BQ molecule 
(in comparison with the phenanthrene). Thus the presence of $\mathrm{N}$ atom causes a deformation of phenanthrene's ring system, and such deformation is topologically extended spreading out not only over the external ring (into which $\mathrm{N}$ atom is incorporated) but also over the central ring of the system (cf. Fig. 1, and also Figs. 2 and 3 in [5]).

The energies of electronic transitions from the ground to the excited states (for the structures optimized by the RHF/6-31G(d) in the ground state) were determined by RCIS/6-31G(d) as well as by the time-dependent DFT method at B3LYP/6-31G(d) level (TD DFT B3LYP/6-31G(d)), and as in many other cases the TD DFT calculations have led to the results much more closer to those experimentally observed than the results of RCIS calculations. Both methods, however, have revealed the same ordering of the excited singlet states, which is: $S_{1}\left(\pi, \pi^{*}\right), S_{2}\left(\pi, \pi^{*}\right)$, and $S_{3}\left(n, \pi^{*}\right)$, with relatively close-lying $S_{1}$ and $S_{2}$ states and with $S_{3}\left(n, \pi^{*}\right)$ at higher energy.

Figure 1 illustrates optimized geometry of BQ molecule in its first excited, $S_{1}$, singlet state, computed in this work with the use of RCIS/6-31G(d) method (as previously all calculations have been carried out using the GAUSSIAN 98 programs [8]). The optimization calculations were verified by the calculations of the vibrational frequencies, which showed that the true minima were obtained.

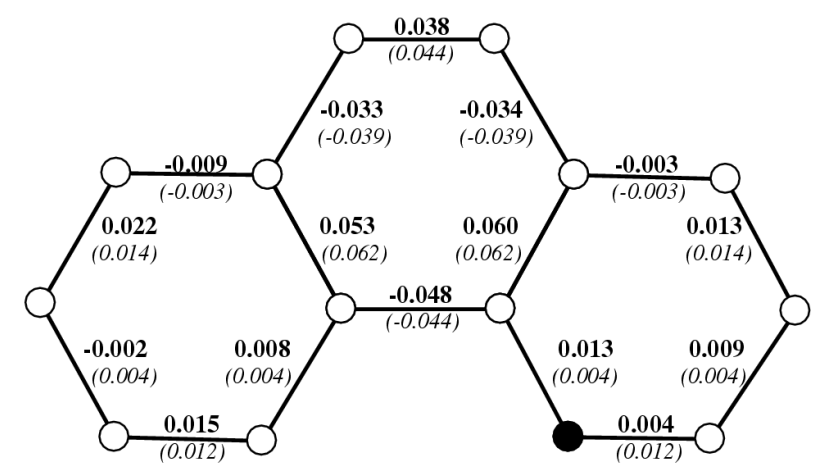

Fig. 1. Optimized equilibrium geometry of the excited $S_{1}$ singlet state of $7,8-$ -benzoquinoline molecule (black dot indicates the nitrogen atom position). Bold numbers are the changes of the length of a given bond relatively to its length in the $S_{0}$ ground state, while the numbers in italics are showing the changes of the corresponding bonds upon excitation of the phenanthrene molecule.

Figure 1 also illustrates how the lengths of corresponding bonds are changing upon transition from the ground to excited state of BQ (indicated by bold numbers). It is seen that upon the excitation of BQ molecule to $S_{1}$ state, the lengths of bonds in the central ring are subject to the largest changes. The numbers given in italics in Fig. 1 are showing relevant changes of the bond lengths upon the 
transition from the ground to the first excited state in a phenanthrene molecule. Comparison of these two sets of numbers is revealing that the changes of bond lengths in the central ring are comparable for both molecules, and that the more pronounced differences can be traced for the bonds in the external rings (not only in the one which contains $\mathrm{N}$ atom, but also in the second one). It is clear that the electronic transition to the first excited state in BQ molecule is not confined to the neighborhood of nitrogen atom, and is delocalized over the entire aromatic rings system, much alike the one in the molecule of phenanthrene.

The TD DFT B3LYP/6-31G(d) computations of electronic transitions for BQ molecule, deliver the energy of its first excited singlet state (of RCIS/6-31G(d) optimized geometry) equal to $30920 \mathrm{~cm}^{-1}$ (and with the oscillator strength of $f=0.035)$. This is ${ }^{1}\left(\pi, \pi^{*}\right)$ electronic state and its energy is much lower than the energies calculated in the previous work (either by RHF/6-31G(d)) or by (DFT B3LYP/6-31G(d)), and of course is also much closer to the experimentally observed transition (see the next section). We should add that, as previously, also the present computations identify the first excited singlet of $\left(n, \pi^{*}\right)$ character as the third $\left(S_{3}\right)$ excited state in the ladder of excited singlets, and with an energy of $c a .4100 \mathrm{~cm}^{-1}$ above $S_{1}$ state.

\section{Analysis of the fluorescence spectrum of jet-cooled BQ molecules}

With the optimized structures of the ground and first excited singlet state one can find the displacement parameters, $\Delta$, for each particular vibrational mode (i.e. the projection of the geometry change between the excited and ground state onto the normal modes of the ground states [9]). This allows us to analyze the vibrational structure of the fluorescence spectrum, provided that the identity of the normal modes is preserved upon $S_{1} \rightarrow S_{0}$ transition.

Reliability of such analysis strongly depends on the resolution of vibrational features in the fluorescence spectrum, and this condition practically excludes most of the experimental data from liquid and/or frozen solutions, crystals and even some low-temperature matrices. Laser induced fluorescence (and fluorescence excitation spectra), observed under cooling conditions of the supersonic jet expansion, are considered to be the best test material for such analysis, although in many cases intensity of dispersed fluorescence is very low and some vibrational activity in $S_{1} \rightarrow S_{0}$ transition may be overlooked.

\subsection{Survey of experimental observations}

Figure 2 shows the laser induced fluorescence (LIF) excitation spectra and dispersed fluorescence spectra observed from the supersonic jet expansion of 7,8-benzoquinoline (seeded in He carrier gas). The figure was compiled with the use of data published in [6], where also all the experimental details can be found. It also illustrates the basic difference between the LIF excitation spectra (recorded for 


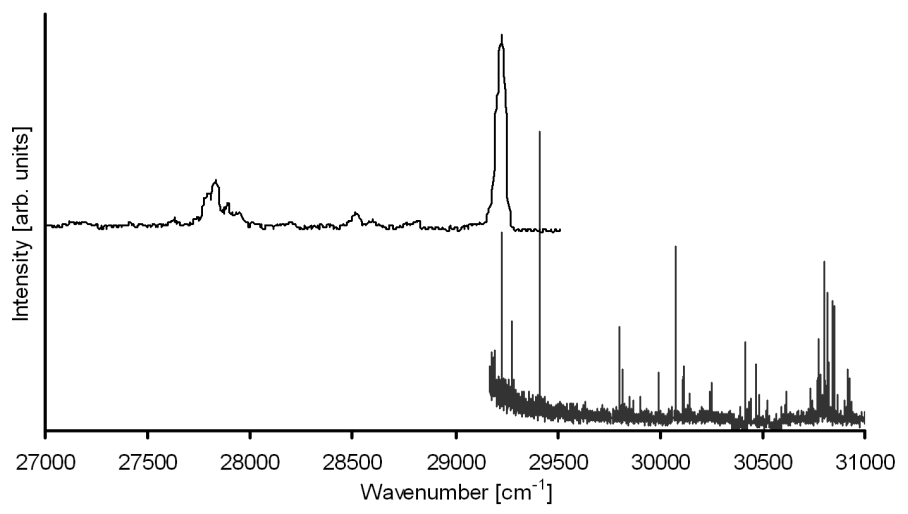

Fig. 2. Excitation spectrum of laser induced fluorescence (bottom-right) and dispersed fluorescence spectrum (top-left) of 7,8-benzoquinoline isolated in the supersonic jet expansion (He carrier gas). The energy of electronic vibrationless origin transition in both spectra is $29226 \mathrm{~cm}^{-1}$. Intensity scales of excitation and fluorescence spectra are not related one to each other (they illustrate only relative intensity distribution within each spectrum).

total fluorescence and with a resolution limited by the spectral width of the laser exciting line) and the dispersed fluorescence spectra (with the resolution limited by the spectral width of the dispersing apparatus).

The LIF excitation spectrum of 7,8-benzoquinoline starts with $(0,0)$ origin band at $29226 \mathrm{~cm}^{-1}$, and in the whole monitored range (down to $\mathrm{ca} .31000 \mathrm{~cm}^{-1}$ ) is very rich of the vibrational structure. The difference between the calculated $\left(30920 \mathrm{~cm}^{-1}\right)$ and experimental energy of the excited $S_{1}$ state (at $29226 \mathrm{~cm}^{-1}$ ) amounts only to $c a .1700 \mathrm{~cm}^{-1}$, which is a very satisfactory test for the method of computations employed in this study.

We notice, for the record, that the origin transition of the LIF excitation spectrum of BQ is shifted by $102 \mathrm{~cm}^{-1}$ toward lower energies (a red shift), relative to the position of the $(0,0)$ origin band in the LIF excitation spectrum of its parent aromatic hydrocarbon, phenanthrene, observed under jet-cooling conditions [7].

Dispersed fluorescence, excited in the $(0,0)$ origin, is very weak (and most of its intensity belongs to the resonant $0-0$ transition). Due to this low intensity of fluorescence, one has to sacrifice the high resolution (such as this in LIF excitation spectrum) and as a result, the vibrational structure of the fluorescence spectrum is diffused (in some regions, also due to the overlap of several vibronic transitions), and besides several (most intense) vibronic transitions, most of the vibrations can be identified only with a large uncertainty. Fortunately, in the case of BQ molecule, this can be overcome to some extent by the observations of single vibronic level (SVL) fluorescence spectrum (see the next section). 


\subsection{Analysis of the vibrational structure of the fluorescence spectrum of $B Q$}

The Table contains some of the computed (RHF/6-31G**) frequencies of the fundamental vibrations of the ground state, which are expected to show up in the $S_{1} \rightarrow S_{0}$ transitions (they are also presented in the form of stick diagram in Fig. 3). All of them are characterized by the non-zero displacement parameter $(\Delta \neq 0)$; vibrations with the displacement $\Delta=0$, i.e. with no change of their equilibrium position between the excited and ground state, should not be observed in the fluorescence spectrum. All vibrations in the Table are of $a^{\prime}$ symmetry.

It comes from the Table that in a frequency range up to $\sim 1600 \mathrm{~cm}^{-1}$ (from the origin) one can expect to see more than 30 active vibrations. Their frequencies should be compared now with vibrational frequencies observed in the fluorescence spectrum.

As we mentioned just above, instead of the origin-excited fluorescence spectrum (given in Fig. 2), we employ for such a comparison an SVL fluorescence spectrum [6], which is observed when the excitation of fluorescence is tuned to the vibrational level of $S_{1}$ excited state which lies $183 \mathrm{~cm}^{-1}$ above the origin (excitation energy of $29226+183=29409 \mathrm{~cm}^{-1}$ ) and which is characterized by the most intense band in the LIF excitations spectrum (see Fig. 2). This spectrum is shown in Fig. 3, and it is clearly seen that the resolution of its vibrational structure is much sharper than in the origin-excited spectrum, and that its vibrational features are definitely more distinct. However, it is also clear that an experimental fluorescence spectrum is a complicated one, with a rich background, under which low-intensity transitions are probably hidden.

Some of the vibrational frequencies which could be extracted from the spectrum accurately enough (with accuracy better than $\pm 5 \mathrm{~cm}^{-1}$ ) are collected in the Table (most of them differ from the frequencies which can be found in Table 2 in Ref. [6], where the preliminary assignment was based on the origin-excited fluorescence spectrum, and thus was less accurate than the frequencies of the SVL fluorescence spectrum used in the present study; cf. discussion in Sec. 3.1).

The comparison of calculated and experimental frequencies listed in the Table shows that they do not coincide exactly, but generally, the deviations of calculated and experimental data are moderate and within the experimental error limits (with a final resolution of $\sim 40 \mathrm{~cm}^{-1}$ in the SVL fluorescence spectrum [6]). At this point, it must be stressed that it is not difficult to achieve a better agreement between computations and experiment under B3LYP calculations of vibrational frequencies. For instance, in such a case two vibrational frequencies, listed in the Table as 687 and $695 \mathrm{~cm}^{-1}$, are becoming 704 and $715 \mathrm{~cm}^{-1}$, respectively, and are getting very close to the experimental frequency of $714 \mathrm{~cm}^{-1}$ (one can also infer from this example that the observed frequency of $714 \mathrm{~cm}^{-1}$ could be just as well the result of the overlap of the two vibrational modes characterized by the displacement parameters of 0.03 and 0.06 ). Such remarks hold probably true also for some other experimental frequencies $\left(1199,1295,1411\right.$ or $\left.1448 \mathrm{~cm}^{-1}\right)$. 
TABLE

Calculated vibrational frequencies $(\nu)$ for $S_{1} \rightarrow S_{0}$ electronic transition in 7,8-benzoquinoline and corresponding displacement parameter $(\Delta)$ of each vibrational mode. For comparison the vibrational frequencies observed in dispersed fluorescence from jetcooled supersonic expansions of 7,8-benzoquinoline (this work) and phenanthrene (frequencies and relative intensities after Ref. [7]) are given.

\begin{tabular}{|c|c|c|c|c|}
\hline \multicolumn{3}{|c|}{ 7,8-Benzoquinoline } & \multicolumn{2}{|c|}{ Phenanthrene } \\
\hline \multicolumn{2}{|c|}{ Calculated } & \multirow{2}{*}{$\begin{array}{c}\text { Experimental } \\
\nu\left(\mathrm{cm}^{-1}\right)\end{array}$} & \multicolumn{2}{|c|}{ Experimental [7] } \\
\hline$\nu\left(\mathrm{cm}^{-1}\right)$ & $\Delta$ & & $\nu\left(\mathrm{cm}^{-1}\right)$ & $I_{\mathrm{rel}}$ \\
\hline 225 & 0.02 & $(247)^{*}$ & $248\left(a_{1}\right)$ & 3 \\
\hline 381 & 0.05 & 410 & $408\left(a_{1}\right)$ & 21 \\
\hline 438 & 0.04 & & $471(\mathrm{~b} 2)$ & 1 \\
\hline & & 467 & & \\
\hline 485 & 0.05 & & $500\left(b_{2}\right)$ & 1 \\
\hline 530 & 0.02 & 557 & $544\left(a_{1}\right)$ & 6 \\
\hline 604 & 0.08 & 619 & $620\left(b_{2}\right)$ & 12 \\
\hline 687 & 0.03 & & & \\
\hline 695 & 0.06 & & & \\
\hline & & 714 & $714\left(a_{1}\right)$ & 12 \\
\hline & & & $731\left(b_{2}\right)$ & 0.1 \\
\hline 815 & 0.06 & & $833\left(a_{1}\right)$ & 13 \\
\hline 859 & 0.02 & 863 & $875\left(b_{2}\right)$ & 20 \\
\hline 978 & 0.05 & & & \\
\hline 986 & 0.10 & & & \\
\hline 1022 & 0.04 & 1026 & $1019\left(b_{2}\right)$ & 1 \\
\hline 1063 & 0.05 & & $1043\left(a_{1}\right)$ & 10 \\
\hline 1076 & 0.07 & & $1065\left(a_{1}\right)$ & 1 \\
\hline 1118 & 0.04 & & & \\
\hline 1133 & 0.01 & & & \\
\hline 1156 & 0.02 & & $1165\left(a_{1}\right)$ & 1 \\
\hline 1181 & 0.03 & & & \\
\hline 1196 & 0.02 & 1199 & $1199\left(a_{1}\right)$ & 1 \\
\hline 1230 & 0.11 & & & \\
\hline 1256 & 0.13 & & $1257\left(a_{1}\right)$ & 1 \\
\hline 1264 & 0.03 & & & \\
\hline & & 1295 & & \\
\hline 1313 & 0.06 & 1342 & $1355\left(a_{1}\right)$ & 40 \\
\hline 1387 & 0.14 & & & \\
\hline 1400 & 0.08 & 1411 & $1423\left(a_{1}\right)$ & 6 \\
\hline 1433 & 0.07 & & $1434\left(b_{2}\right)$ & 6 \\
\hline 1439 & 0.06 & 1448 & $1450\left(a_{1}\right)$ & 26 \\
\hline 1483 & 0.06 & & & \\
\hline 1512 & 0.06 & 1521 & $1526\left(a_{1}\right)$ & 1 \\
\hline 1585 & 0.27 & 1568 & $1580\left(a_{1}\right)$ & 2 \\
\hline 1606 & 0.16 & 1604 & $1613\left(a_{1}\right)$ & 18 \\
\hline 1619 & 0.24 & & & \\
\hline 1635 & 0.16 & & & \\
\hline
\end{tabular}




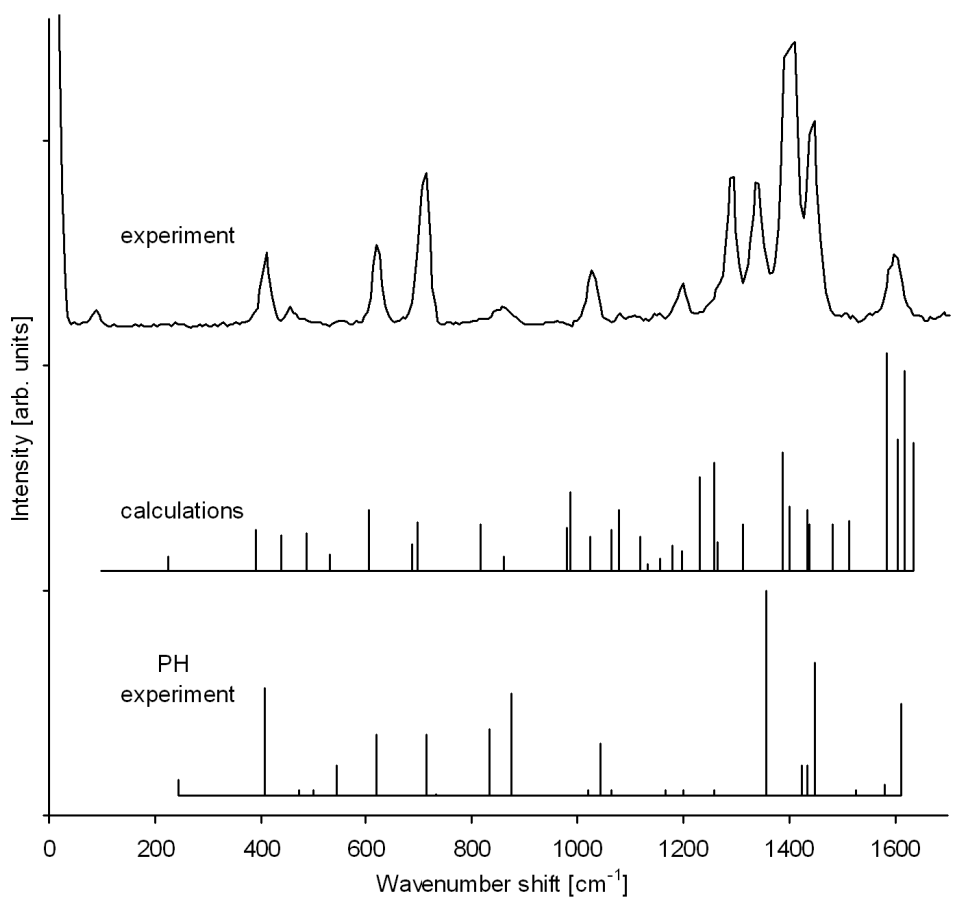

Fig. 3. (Top) Single vibronic level fluorescence spectrum of 7,8-benzoquinoline observed after excitation with an excess energy of $183 \mathrm{~cm}^{-1}$ above the origin transition (excitation energy $\left.29226+183=29409 \mathrm{~cm}^{-1}\right)$. (Middle) Calculated vibrational frequencies of the fluorescence spectrum (for details see the text). (Bottom) Dispersed fluorescence spectrum of jet-cooled phenanthrene (PH) molecule (compiled after data of [7]). All spectra are displayed as the energy shifts relative to the energy of the origin transition.

The above examples and remarks are once again illustrating the well-known dependence of the results of calculations on the method employed. With this in mind, we believe that in the case of fluorescence spectra, i.e. $S_{1} \rightarrow S_{0}$ vibronic transitions (with $S_{0}$ state being a terminal state), it is more appropriate and physically more reliable to stick to the RHF/6-31G** methods, which allows for the optimization of the ground and as well as the excited states involved in the transitions under consideration. The search for a better agreement of calculated and experimental data should be assured by a higher resolution of the fluorescence (and elimination of the scattered laser light in the spectrum).

In view of the conclusions of Sec. 2 concerning similarities and differences between the $S_{1}$ excited state of BQ and phenanthrene, it is expedient to compare vibrational features of the fluorescence spectrum of BQ with those which were observed in the fluorescence spectrum of phenanthrene molecules in the supersonic expansion [7]. Relevant vibrational frequencies present in the phenanthrene fluorescence spectrum, are listed in the Table (together with their relative intensities) 
and given as a stick diagram in Fig. 3. The first and most noticeable difference between the both spectra is a relatively poor vibrational structure of the fluorescence of phenanthrene in comparison to the spectrum of BQ. Secondly, the Table reveals that there are vibrational frequencies in both spectra that exhibit one-to-one correspondence (e.g. 714 and $1199 \mathrm{~cm}^{-1}$ vibrations) or near correspondence (e.g. 410 and 408, 619 and 620, 1026 and $1019 \mathrm{~cm}^{-1}$ and some others). And furthermore, there are some very clear differences of the intensity for given vibrational transitions in the fluorescence spectra of both molecules.

The investigations of mid-infrared ground-state spectra of polycyclic aromatic nitrogen heterocycles in low-temperature inert matrix (argon) have led to the general conclusion that the presence of nitrogen atom in some aromatic N-heterocyclic compounds (among them in 7,8-benzoquinoline) delivers characteristic, intense vibrational mode of $\sim 1400 \mathrm{~cm}^{-1}$, which is absent in the IR spectrum of the parent aromatic hydrocarbon [11]. We notice that such a vibrational feature is observed in the fluorescence spectrum of jet-cooled BQ with the nominal frequency of $1411 \mathrm{~cm}^{-1}$, and as it comes out from the computations, there are two relatively strong vibrations $\left(1387\right.$ or $\left.1400 \mathrm{~cm}^{-1}\right)$ that can contribute to the observed vibrational band (cf. Fig. 3 and Table). However, the above general conclusion, inferred from the IR spectra, may not necessarily be applicable to the fluorescence spectrum, in which vibrational activity of a given mode is governed and controlled by its form and by its equilibrium geometry changes upon excitation. And thus our calculations are suggesting (and this is in accordance with experimental observation) that 1230 and $1256 \mathrm{~cm}^{-1}$ vibrations should be active in the fluorescence spectrum of BQ, although in the ground-state IR spectrum they were not observed [11], and $1257 \mathrm{~cm}^{-1}$ mode is of very low intensity in the fluorescence spectrum of phenanthrene molecule [7].

All the above-discussed vibrational modes are executed with participation of the whole molecule (with its all molecular bonds engaged), and as such are reflecting the symmetry break caused by the introduction of the nitrogen atom into the aromatic rings skeleton of the phenanthrene.

\section{Summary}

The results of calculations carried out in this work lead to the conclusion that the symmetry break, caused by the substitution of $\mathrm{N}$ atom into the ring system of phenanthrene molecule, is rather moderately reflected by the changes of the bond lengths in the first excited singlet state of the 7,8-benzoquinoline molecule, and is not localized or confined to the neighborhood of nitrogen atom. This conclusion was further confirmed by the results of the vibrational structure analysis of fluorescence spectrum of BQ observed under supersonic jet-cooling conditions. It has turned out that all prominent vibrations which become active in this spectrum (and which are of rather low intensity in the fluorescence spectrum 
of phenanthrene), are related to the above-mentioned symmetry break caused by the introduction of nitrogen atom. Some of the other vibrations present in the spectrum of $\mathrm{BQ}$ exhibit near-correspondence with vibrations observed in the phenanthrene's fluorescence spectrum.

We expect that the more detailed description of the effect of symmetry breaking in monoazaphenanthrenes and its role in the observed differences in the photophysics of this molecular family should become possible with the results of subsequent analysis of fluorescence spectra of phenanthridine, which is now in the course of study.

\section{Acknowledgment}

We gratefully acknowledge a partial financial support of this work by the State Committee for Scientific Research under project 5 P03B 13620.

\section{References}

[1] R.S. Becker, Theory and Interpretation of Fluorescence and Phosphorescence, Wiley Interscience, New York 1969.

[2] E.C. Lim, in: Excited States, Ed. E.C. Lim, Vol. 3, Academic Press, New York 1977, p. 305.

[3] L.A. Diverdi, M.R. Topp, J. Phys. Chem. 83, 3477 (1984).

[4] M. Norek, J. Dresner, J. Prochorow, Acta Phys. Pol. A 104, 425 (2003).

[5] I. Deperasińska, J. Prochorow, Acta Phys. Pol. A 104, 601 (2003).

[6] J. Prochorow, I. Deperasiska, Y. Stepanenko, Chem. Phys. Lett., in press.

[7] J.A. Warren, J.M. Hayes, G.J. Small, Chem. Phys. 102, 323 (1986).

[8] Gaussian 98, Revision A.9, M.J. Frisch, G.W. Trucks, H.B. Schlegel, G.E. Scuseria, M.A. Robb, J.R. Cheeseman, V.G. Zakrzewski, J.A. Montgomery Jr., R.E. Stratmann, J.C. Burant, S. Dapprich, J.M. Millam, A.D. Daniels, K.N. Kudin, M.C. Strain, O. Farkas, J. Tomasi, V. Barone, M. Cossi, R. Cammi, B. Mennucci, C. Pomelli, C. Adamo, S. Clifford, J. Ochterski, G.A. Petersson, P.Y. Ayala, Q. Cui, K. Morokuma, D.K. Malick, A.D. Rabuck, K. Raghavachari, J.B. Foresman, J. Cioslowski, J.V. Ortiz, A.G. Baboul, B.B. Stefanov, G. Liu, A. Liashenko, P. Piskorz, I. Komaromi, R. Gomperts, R.L. Martin, D.J. Fox, T. Keith, M.A. Al-Laham, C.Y. Peng, A. Nanayakkara, M. Challacombe, P.M.W. Gill, B. Johnson, W. Chen, M.W. Wong, J.L. Andres, C. Gonzalez, M. Head-Gordon, E.S. Replogle, J.A. Pople, Gaussian Inc., Pittsburgh (PA) 1998.

[9] A. Bérces, M.Z. Zgierski, in: Computational Molecular Spectroscopy, Eds. P. Jensen, P.R. Bunker, John Wiley and Sons Ltd., Chichester 2000, p. 109.

[10] J. Prochorow, M. Norek, B. Kozankiewicz, to be published.

[11] A.I. Mattioda, D.M. Hudgins, C.W. Bauschlicher Jr., M. Rosi, L.J. Allamandola, J. Phys. Chem. A 107, 1486 (2003). 